Research Article

\title{
Empirical Study on Theories and Techniques of Adolescent Physical Health Promotion under the Background of Big Data
}

\author{
Haolun Xu \\ Department of Teacher Education, Nanchong Vocational and Technical College, Nanchong 637000, Sichuan, China \\ Correspondence should be addressed to Haolun Xu; xhl321zxy@163.com
}

Received 1 July 2021; Revised 7 August 2021; Accepted 18 August 2021; Published 27 August 2021

Academic Editor: Sang-Bing Tsai

Copyright (C) 2021 Haolun Xu. This is an open access article distributed under the Creative Commons Attribution License, which permits unrestricted use, distribution, and reproduction in any medium, provided the original work is properly cited.

\begin{abstract}
In order to make my country's youth health management more scientific, more standardized, and more effective, it is necessary to conduct in-depth research on the management of youth physical health promotion. Through the investigation and analysis of the physical health data of adolescents in my country, this paper proposes that the results of health intervention training as part of the empirical research on the construction of adolescent health big data management service system can effectively improve the relationship hypothesis of the physical health of adolescents and by selecting the example of our country's "Adolescent Physical Health Data Analysis in 2020" for regression analysis. The research results show that the theory of adolescent physical health promotion can improve the physical health of adolescents by interfering with students' physical exercise. In the processing of data, GBDT is suitable when the training set is relatively large, and as the sample size increases, the accuracy rate can reach $79.79 \%$. In terms of the classification accuracy of male sitting forward bending promotion, the accuracy of the RF method is higher than that of GBDT. In terms of the promotion classification effect of boys' $1000 \mathrm{~m}$ running, the RF method achieved the highest promotion accuracy rate of $77.62 \%$. In the male pull-ups to promote the classification effect, when the proportion of the training set is $60 \%$, the RF method gets the highest accuracy rate, which is $92.04 \%$. The results of the classification effect for girls standing long jump promotion show that the classification accuracy rate for girls standing long jump promotion is between $51 \%$ and $56 \%$. When the training set is less than $60 \%$, the RF method is the best, the highest is $53.93 \%$, and the rest is the GBDT method, the highest is $55.46 \%$; in Macro-F1, the RF and GBDT indicators have their own advantages. In the promotion of the classification effect on the final fitness level of girls, the accuracy rates of RF and GBDT methods range from $90 \%$ to $96 \%$, and the accuracy rates of the NN method range from $80 \%$ to $87 \%$; when the practice rate reaches $80 \%$, the GBDT method achieves the highest accuracy rate of 95.06\%; on the Macro-F1 index, the GBDT method is obviously the best.
\end{abstract}

\section{Introduction}

Knowledge of physical health plays an important role in understanding physical fitness. An important goal of highquality physical education is to impart sports knowledge to young people to promote exercise. Lack of physical health knowledge is one of the main factors leading to adolescents' lack of physical exercise. Knowledge of physical health is considered to be a core element in the field of cognitive sports. Together with the knowledge of healthy lifestyle and the value of physical activity, it is considered to be the three important components of physical literacy, and it is the basis and prerequisite for achieving physical literacy. However, the physical health of young people is still showing a downward trend. Most young people are still on the pass and fail levels, and the proportion of young people who reach good and excellent is small.

Juul et al. separated health promotion from disease prevention and tried to change behavior and unhealthy lifestyles by improving education, policies, and the environment, thereby promoting physical health [1]. Beaton and Freeman interpreted health promotion as "a science and art that helps people change their living habits to achieve an ideal state of health. The ideal state of health should be to achieve physical, emotional, social adaptation, and spiritual and intellectual balance" [2]. Based on the concept of health promotion at different stages, Borhani et al. believes that health promotion is a combination of natural science, health 
science, and behavioral science knowledge and seeks to improve the lifestyle including physical activity, eating habits, and mental state. Harmony with the entire environment to improve the overall strategy of life quality [3]. Bnnelycke et al. can see from the concept and development of health promotion that there are many disciplines and complex theoretical foundations for the study of adolescents' physical health problems [4]. Winter et al. believe that the theoretical basis of the research on the promotion of adolescent physical health mainly includes social learning theory, life course theory, behavior change theory, and social ecology theory [5].

Burau et al. proposed that the promotion of adolescent physical health must be guided by a scientific and systematic top-level design to guide the long-term development direction. Adolescent health promotion must always emphasize the organic connection and convergence of internal elements to ensure the unity and harmony of theory and practice [6]. Tiitinen et al. emphasized that individuals complete the matching of personal behavior and environment through a certain group and social relationship [7]. $\varnothing$ sterlie et al. proposed that the promotion of adolescent physical health requires systematic theoretical guidance as the foundation to support its sustainable development and improvement [8]. Vogel proposed that although BMI is the most widely used to measure physical fitness in the world, there are still controversies, mainly focusing on whether BMI is suitable for the weak physical fitness standards of different racial/ethnic populations, and there is a lack of systematic theoretical analysis [9]. Hales et al. proposed a new quantitative perspective to conduct physical health research on Americans of different races and believe that the physical promotion of different races is very different [10]. Although the above studies have achieved certain results in terms of physical health, they have not compared the actual effects of the proposed theories on young people, and there is also a lack of data testing in the context of big data.

This article focuses on the physical health of adolescents and is dedicated to the study of multidimensional data synthesis of adolescent physical fitness models. Firstly, it collects the data of Chinese adolescents from 2016 to 2020. Secondly, it conducts empirical analysis of data through detailed data mining and then uses machine learning algorithms to build models to drive different levels of test items so as to warn young people in need, finally compare the promotion performance effects of different models on the dataset, and promptly promote and intervene in young people with poor physical fitness. Taking linear combination exercise rules as exercise strategy, applying extreme big data physical fitness promotion theory to get regression exercise model so that it can generate exercise rule weight combination suitable for physical condition and achieve multiobjective optimization of physical fitness. This paper establishes a physical fitness data model, combined with co-integration analysis and error correction methods to empirically analyze the impact of physical fitness promotion theories and technologies on the physical health of adolescents so that the results of the analysis are more stable and reliable.

\section{Theories and Techniques of Promoting Adolescent Physical Health}

2.1. Status Quo and Promotion Methods of Youth Physical Exercise. The sports infrastructure and sports facilities of different countries are very different, but they have a lot in common. Just like every school in Japan has a baseball field and every school in the United States and Canada has a football field, there are representative schools that young people love $[5,11]$. Seasonal events such as ice and snow in the north and water sports in the south have their own strengths. Some local courses are set aside for local or school choices. Provinces and cities with conditions will choose national curriculum standards. If there are no conditions, local self-control can be implemented [12]. Regarding the content of health education, it is necessary to further specify who teaches and how to teach, so as to facilitate assessment and evaluation, thereby reducing the confusion of physical education teachers [13]. If the current national physical education curriculum standards remain the status quo and lack the cooperation and effective connection of local and school education functional departments at all levels, experts, scholars, and majority of physical education teachers will go their own ways, and the training goals and training content cannot be deeply integrated, and there is no close connection [14]. The main content of the physical health promotion theory (PHPT) proposed in this article is diversified physical education curriculum models, streamlined groupings, and unit exercises.

\subsubsection{Advocate Diversification of Sports Curriculum Models.} The main subject characteristic of physical education curriculum is skills, which determines that physical education curriculum has many different styles and genres, and the diversification of physical education curriculum entity models is necessary and possible [15]. In different countries at different times, there are generally three ways to design and select physical education curriculum models: one step, full delegation, and step by step. Our country's physical exercise program period is one step in place, and physical education teachers only need to solve the problem of exercise [16]. Some provinces, cities, and regions in our country have inadequate supervision and went directly from the first step to the third or fourth step. Without the support of the second or third step, even serious, responsible, and experienced physical education teachers will be helpless [17]. The basic physical education in the past 20 years has proved that many physical education teachers are still struggling in determining and designing physical education curriculum models. In the application of the curriculum model, it is not possible to make absolute and simplistic, but also to consider relevant factors such as curriculum objectives, youth development level, subject nature, training conditions, and teacher possibility, so as to achieve the expected results. Regardless of the curriculum model adopted, copying and imitation are not advocated [18, 19]. Curriculum has a model, but there is no fixed model. Trying to design a model regardless of sports events, different characteristics of adolescents, and other related factors is 
tantamount to blindly prescribing to cure all diseases. As a result, there is a tendency toward standardization and modelization. Physical education teachers have their own ways, and there is never a time for convergence [20]. Only by implementing the diversification of physical education curriculum models, the coexistence of various styles of physical education curriculum models, and not guiding the practice of physical education curriculum in a fixed mode, can the initiative and creativity of physical education teachers be further brought into play [21].

2.1.2. Reasonably Arrange Group Exercises and Unit Exercises. Group work usually refers to class work. The form of classroom teaching helps to expand the scale of teaching, but for physical education, the main disadvantage is that it is not convenient to teach students in accordance with their aptitude, and they cannot treat young people's physical fitness and uneven physical abilities differently, which affects their personality development [22]. With the in-depth advancement of the current physical education curriculum reform, physical training in groups according to different curriculum models, school physical education curriculum settings, school venues and equipment, and teacher qualifications, there are classes according to gender, physical fitness, and physical fitness. There are many forms such as sports performance classification and selection of sports events [23]. On this basis, elementary school physical education sometimes requires two or more courses and group exercises in the class. The group exercises are based on the actual situation of the young people. Group exercises are carried out according to the actual situation of young people, they can be set to rotate in groups or nonrotating in groups, group by isomorphic design, and group by heterogeneous design [24]. Various forms of group exercise are helpful to the organization and management of physical exercise. There is little difference in the interests and sports level of young people during group exercise, which can easily meet the needs of young people and improve the quality of exercise. No matter what kind of grouping exercise organization form is adopted, scientific argumentation must be carried out and cannot be artificially divided into groups, which will affect the completion of course objectives and academic performance. Group exercise needs to maximize the interests of young people and optimize the effect of exercise $[25,26]$. Unit exercise, as a relatively independent exercise process as a whole, is closely related to group exercises and is an important part of completing the course implementation. When constructing unit exercises, we must first select textbooks reasonably to meet the characteristics of the subject, the physical and psychological characteristics of adolescents, and their actual needs and plan the overall arrangement of class hours and exercise content, as well as the reasonable combination of textbooks, including the goals and objectives of each class [27]. Young teenagers are easily distracted. Primary school physical exercises are suitable for compound textbook units. One lesson can use two or more exercise contents for rotation exercises to mobilize young people's interest in sports. When using the compound exercise unit in middle schools, it should also consider the promotion relationship between subjects, including the comprehensive exercise of upper limbs and lower limbs, exercise techniques, physical fitness, and other aspects of teaching materials. In the advanced stage of middle school, if conditions permit, a single textbook unit can be used to cultivate special sports for young people and form a stable sports hobby. A single textbook unit does not exclude some supplementary textbooks to make up for the shortcomings of the main textbooks, such as compensatory exercises for physical fitness and expansion exercises for preparation and recovery. A physical education class often only teaches a single technique, while a unit needs to teach a complete series of combined techniques for a certain project. The exercise goal actually governs the goal of several classes, and the arrangement and combination of exercise content in several classes need to be considered. In addition, physical education courses should be closely integrated with other subject courses to form a comprehensive exercise unit and continue to play the function and role of physical education courses. For example, the combination of field trips, exercise internships, and holiday camps (carrying out crosscountry running, cycling, skiing, skating, etc.) to carry out a variety of sports competitions has broad prospects and space for development.

\subsection{Adolescent Physical Data Evaluation Model}

(1) Decision matrix for juvenile physical health standards:

The information of indicators $\mathrm{C} 1$ and C2 is represented by precise numbers, while the information of indicators $\mathrm{C} 3-\mathrm{C} 11$ can only be represented by qualitative semantics. In order to establish a standardized matrix, using a given method, the decisionmaking information of the two types of adolescents' health standards are expressed as visual blur numbers. For the decision-making information of indicators $\mathrm{C} 1$ and $\mathrm{C} 2$, the physique health expert group firstly gives the expected interval of $\mathrm{C} 1$ and $\mathrm{C} 2$ in each stage based on the physical health status of adolescents and then calculates the intuitionistic fuzzy number of the decision information of each adolescent physique health standard of C1 and C2 indicators. For the semantic information corresponding to the indicators $\mathrm{C} 3-\mathrm{C} 11$, find the intuitionistic fuzzy number corresponding to each semantic information in the table.

(2) Decision matrix of weighted physical fitness standards at each stage: 
First, use formula (1) to obtain the intuitionistic fuzzy information entropy of each stage C1-C11, respectively, and then calculate the weight of each stage index C1-C11. Finally, based on Definition 3 and the required weights, a weighted decision matrix for each stage is constructed.

(3) The positive and negative ideal solutions of physical fitness standards at each stage:

First, based on the size of the defined comparison information, select the positive and negative ideal solutions according to the rules. Then, for cost-based indicators, the positive ideal solution will select the minimum value of the stage candidate data, and the negative ideal solution will select the maximum value of the stage candidate data; for the benefit index, the positive ideal solution will select the maximum value of the stage candidate data value, and a negative ideal solution will select the minimum value of the candidate physique data at the stage. Among the decision-making indicators proposed in this paper, $\mathrm{C} 1$ and $\mathrm{C} 2$ are cost-based indicators, and $\mathrm{C} 3-\mathrm{C} 11$ are benefit-based indicators. The ideal formula for stage positive and negative is as follows:

$$
\begin{aligned}
& P\left(d_{i}, w_{j}\right)=P\left(d_{i}\right) P\left(w_{j} \mid d_{i}\right), \\
& P\left(w_{j} \mid d_{i}\right)=\sum_{k=1}^{K} P\left(w_{j} \mid z_{k}\right) P\left(z_{k} \mid d_{i}\right), \\
& \lambda(A i, A j)=\left[\log \left(\frac{\left|x_{A_{i}}-a_{A_{j}}\right|}{w_{A_{j}}}\right), \log \left(\frac{\left|y_{A_{i}}-y_{A_{j}}\right|}{h_{A_{j}}}\right), \log \left(\frac{w_{A_{i}}}{w_{A_{j}}}\right), \log \left(\frac{h_{A_{i}}}{h_{A_{j}}}\right)\right] .
\end{aligned}
$$

(4) The closeness of each physical fitness data to the ideal solution in the stage:

First, calculate the distance from each physical fitness data to the positive and negative ideal solution in the stage according to the formula, and then, calculate the closeness of each physical data to the ideal solution in the stage according to the formula. The closeness of each physical data in stage 1 is

$$
\begin{aligned}
& y=\beta X+\varepsilon, \\
& \varepsilon=\lambda W+\xi
\end{aligned}
$$

The closeness of each physical fitness data in stage 2 is

$$
\begin{aligned}
\phi & =V \times \frac{n}{Q}+\frac{Q}{(D / 2) 2 \times \pi \times 3600}, \\
(I n-\alpha W) y & =(I n-\alpha W) X \beta+\varepsilon .
\end{aligned}
$$

The closeness of each physical fitness data in stage 3 is

$$
\begin{aligned}
f_{R}^{A_{i}} & =w_{G}^{A_{i} A_{j}} \cdot V, \\
w_{G}^{A_{i} A_{j}} & =\max \left\{0, W_{G} \cdot \varepsilon\left(f_{G}^{A_{i}}, f_{G}^{A_{j}}\right)\right\} .
\end{aligned}
$$

(5) Feasible combination of physical fitness data:

First, calculate the number of potential physique data combinations. The number of candidate physique data at each stage is 4,3 , and 5 , respectively. The number of physique data required for each stage is 1 . According to the theory of physical fitness evaluation, the number of potential physique data combinations is $N=4 \times 3 \times 5=60$ pieces. Therefore, after removing the combination of physical data that contains any of the above four pairs of physical data, the number of possible physical data combinations is 39 .

(6) Collaboration degree of feasible physical data combination:

Physical fitness monitoring is based on the synergy of historical data and physical fitness data, respectively, gives the influence factors of the physical data of stage 1 on the physical data of stages 2 and 3 and the influence factors of physical data of the stage 2 on the physical data of stage 3 . Then, use the formula to calculate the degree of coordination between the physical data of Phase 2 and the physical data of Phase 1 and the degree of collaboration of the physical data of Phase 3 under different combinations.

(7) The final evaluation result of each feasible physical data combination:

According to the physical data combination, to meet the multistage requirements for physical data based on the CoPS youth physical health, construct the CoPS green selection index system, and at the same time, consider the synergy between the multistage physical data from the characteristics of the CoPS complex system to obtain the final physical health evaluation result.

2.3. Physical Health Big Data Service Model. The construction of a data-driven physical health big data service complex system as a whole follows three basic principles: (1) covers 
smart data, user needs, smart technology, big data physical health, physical health workers, big data service platforms and physical health analysis methods, seven key elements; (2) reflecting the four characteristics of data multisource, demand sensitivity, technical intelligence, and service scenario; (3) realizing the three cores of demand big data perception, physical health service big data research, and user service intelligent push function, and its formula is as follows:

$$
x_{i}=\sum_{j=1}^{n} \omega_{i j} y_{j}-\theta_{i} .
$$

Among them, the distance to the service system area is the physical standard deviation, which is an important regularization parameter in the GWR model. The size of the system will directly determine the physical health change scale feature of the solution coefficient, and the bandwidth corresponds to a fixed type (that is, a fixed distance threshold) and variable type (that is, the preset distance value corresponding to the $\mathrm{Nth}$ nearest field is used as the bandwidth value of each solution point), and this paper adopts the variable type physique data model. When solving the BMI model, the bandwidth needs to be optimized. The cross-validation method or the BMI method can be used. When solving the bandwidth of the BMI model, the corrected version of the BMI method is generally used, and the expression is as follows:

$$
\begin{aligned}
\operatorname{BMI}(b) & =2 n \ln (\sigma)+n \ln (2 \pi)+n\left\{\frac{n+\operatorname{tr}(S)}{n-2-\operatorname{tr}(S)}\right\}, \\
F & =\sum_{i=1}^{k} \sum_{q \in D_{i}}\left|q-n_{i}\right|^{2}, \\
W(T) & =K(y(T-1), u(T-d-1)),
\end{aligned}
$$

where $F$ represents the estimated variance, $S$ represents the data range matrix of physical fitness, and $W(T)$ represents the trace of the matrix. The construction of a complex system of the physical health big data service system has three main lines of thinking: (1) based on multisource heterogeneous massive data resources, realize the core functions of physical health big data services; (2) between the constituent elements of the physical health big data service model, mutual coordination then constitutes a complete physical health service ecosystem; (3) it reflects the main characteristics of the physical health big data service model. Since the BIM software lacks the physique health family model required by this complex system, it is necessary to determine the data scale of the required model based on the preliminary complex system big data type investigation, analysis, and formula calculation and determine the number of models based on the formula. For example, the SJG diagonal flow physical examination model in the physical health big data service system model is established as an example: according to the design specifications, indoor health data functional partitions and usage requirements, the indoor physical health monitoring index is determined to be $Q$, and the accuracy of the complex system is $D$, which can be calculated as health index $v$, namely,

$$
v=Q\left(D_{2}\right) 2 \times \pi \times 3600 .
$$

2.4. Physical Health Promotion Theory and Technical Implementation Model. The optimization of the physical health big data service system involves cross-professional optimization. It is no longer a simple system. First, determine the key target performance of the system and list it as the "refactoring pole." They are usually the core performance and functions of the big data service system. Its mathematical representation is as follows:

$$
U=\sum_{i=1}^{g}\left\{P_{i} \mid \sum_{j=1}^{k} p_{j}^{(i)}\right\} .
$$

The role of physical health knowledge in promoting physical activity can also be explained by TPB theory. According to TPB theory, an individual's intention to participate in physical activity is a direct promotion indicator of individual physical activity participation, which contains three decisive factors. The importance of physical activity knowledge and participation is that it is related to the factors of TPB attitude. To be clear, knowledge affects attitudes and thus the purpose of behavior; assuming that physical activity, health knowledge, and health benefits are the basis of physical activity participation, then improving and developing physical health knowledge should be the first step in establishing healthy physical activity behavior. Therefore, behavioral control is also one of the mediating variables that mediate the relationship between physical health knowledge and physical activity behavior. In the case of integrated reconstruction of vibration isolation and shock resistance, three reconstruction poles are determined:

$$
U=\left\{P_{1}\left|D, L, f_{2}, Q, d, l, P_{2}\right| f_{1}, \mu, P_{3} \mid N, M, I\right\} .
$$

In the formula, $P 1$ is the overall pole, $2 P$ is the health pole, and $3 P$ is the physical evaluation pole. Here, $P 3$ is listed as the comparison object, but it can also be other. Because it is iterative optimization, the initial plan is not particularly important; the acceptable plan is marked as $U$. Decompose the physical elements and redesign the architecture; then, mark the initial parameter scheme as (0) $C$, change the parameters, obtain the improvement scheme, and compare and summarize the optimization direction, until satisfied. The system is an envelope relationship to the subsystem, so subsystem optimization can use all mature technologies, theories, and research results. For example, pure structural optimization can use structural dynamics methods to establish objective optimization functions to carry out optimization analysis; difficult to express or immature combination of overall expression or complex system experience decision-making, and they are finally presented quantitatively on the data table, which is for the complex system decision-making of the physical health big data service system. In fact, the comparison of advantages and disadvantages is already obvious. The key performance parameters in the reconstruction pole can be replaced, but the most important evaluation parameters at the moment 
are always retained, leading to a "chemical reaction" to generate one or more performance/function improvements. The general expression of the basic GWR model is as follows:

$$
\begin{aligned}
I R & =\sum_{S-1}^{U} \sum_{d-1}^{K} f_{s}, D V_{s}, d, \\
y_{i} & =\beta\left(u_{i}, v_{i}\right)+\sum_{j=1}^{p} \beta_{j}\left(u_{i}, v_{i}\right) x_{i j}+\varepsilon_{j} \beta_{j} .
\end{aligned}
$$

Among them, $\left(u_{i}, v_{i}\right)$ are the coordinates of the regression analysis point, $X_{j}(j=1,2, \ldots)$ is the independent variable, and the regression parameter is about the physical health function, that is, it is estimated to quantitatively reflect the heterogeneous characteristics of physical health by evaluating the physical health status quantitatively. The MBY model uses the linear weighted least element method to decompose the model at $i$ in any regression analysis. The formula is as follows:

$$
\beta_{j}=\left(X^{T} W_{j} X\right)^{-1} X^{T} W_{j} Y
$$

The matrix $X=\left(x_{i j}\right)_{n \times(p+1)}$ is the independent variable sampling matrix (the value of the first column is 1 ), $Y=$ $(y 1, y 1, \ldots, Y n)^{T}$ is the dependent variable sampling matrix, and $W_{i}$ is the diagonal matrix, where the diagonal element $w i k(k=1, \ldots, n)$ represents the value of each observation at the regression analysis point $i$. The weight is generally calculated by the kernel function. Finally, the migration operator is executed after determining the migration candidate solution in the migration subsystem, which is defined as

$$
\begin{array}{ll}
x_{i k}(s) \longleftarrow x_{j l}(s), & \\
\sigma_{i k j l}= \begin{cases}\frac{n}{\Delta_{i k j l}} \sqrt{\sum_{s=1}^{n}\left(x_{i k}(\varepsilon)-x_{j l}(\varepsilon)\right)^{2} \Delta_{i k j l}(\varepsilon),} & \Delta_{i k j l}>0, \\
0, & \Delta_{i k j l}<0 .\end{cases}
\end{array}
$$

Among them,

$$
\Delta_{i k j l}=\sum_{\delta=1}^{n} \Delta_{i k j l}(\varepsilon) .
$$

2.5. Relationship Hypothesis of the Influence of the Adolescent Physical Health Promotion Theory on Adolescents. All mass data related to physical fitness and health can be called health big data. At this stage, with the continuous acceleration of informatization in the field of physical fitness, the types and scale of health data are increasing exponentially. The difference from big data in other industries is that, in addition to the $5 \mathrm{~V}$ characteristics of general big data, physical big data also has the characteristics of integration, dissemination, and intelligence. Based on this, the following hypotheses are proposed.
Hypothesis 1. Targeted health intervention training and using the results of health intervention training as part of the empirical research part of the construction of adolescent health big data management service system can effectively improve the physical health of adolescents. Many adolescents lack physical exercise, do not even pay attention to the results of physical examinations, and do not treat physical examination results and academic performance at the same level. This requires all parties to guide young people to change their thinking and pay attention to their physical health.

Hypothesis 2. Exercise can enhance the individual's sense of control and self-efficacy, produce positive psychological changes, and transfer the individual's bad emotions, thereby increasing the interest of young people in improving physical health. The current evaluation mechanism does not make young people feel the pressure of physical health and actively pay attention to their physical health and participate in exercise. Currently, there is a lack of an effective early warning system. If early warning and monitoring can be made, young people can realize that their physical condition is not good enough, and they may fail the physical test and have enough time to improve, and the downward trend should be able to change.

Hypothesis 3. The idea and method of collaborative governance intervene in the field of youth physical exercise governance, with good adaptability and pertinence. The importance of physical exercise has been unanimously recognized in the academic circles, but practical problems such as poor participation in youth physical exercise and low management effectiveness have been unresolved. The lack of scientific and effective governance theoretical guidance is the key reason. It is a wise choice to involve the idea and method of collaborative governance in the field of youth physical exercise governance.

\section{Construction of an Empirical Research Model of Theories and Techniques for Adolescent Physical Health}

3.1. Data Sources for Empirical Analysis of Physical Fitness. This article takes the 2016-2020 General College Adolescent Physical Test Data as the research object. Among them, there are 11,927 male data and 4854 female data. In the process of research, we must fully consider the objective basic conditions. Before conducting the research, the feasibility of the experiment must be investigated and studied. And, on the basis of fully satisfying relevant theories, the research objects are formed. In terms of sports training, the main research content in the course of research on this course is sports training, related methods, and theories of physical education.

\subsection{Empirical Research Data Analysis Steps}

(1) Physical test data processing:

In the face of massive amounts of data, use the matplotlib library in python as a tool to draw the data 
distribution of each data item (including discrete variables and numerical variables). Through the data distribution, the degree of concentration and dispersion of the data can be seen. Through the comprehensive grade data distribution diagram, it is easy to analyze that except for weight scores, and most people have the most normal grades and pull-ups failing. Other test items are the most passing grades, and the proportion of good and excellent people is very small. The sample distribution is extremely uneven. In addition, we use descriptive statistics to do exploratory analysis of the data and know the basic statistical values of each variable, including the mean, standard deviation, variance, and mode.

(2) Physical feature extraction and processing:

After a simple exploratory analysis of the dataset in the data understanding stage, the data is further processed to provide reliable data for the next machine learning algorithm. Experiments have verified that there are no missing or duplicate values in the dataset. Then, normalize it to extract a new feature body mass index (weight/height). Finally, the discrete variable or categorical variable is processed by the ne-hot encoding method.

(3) Model processing of physique data:

After the data is manipulated in the data preparation stage, the dataset meets the requirements and can be directly used for model building and training promotion. Based on the random forest algorithm (RF) model to achieve the RF algorithm flow and the gradient boosting tree algorithm- (GBDT-) based model to achieve the GBDT algorithm is based on the boosting algorithm, essentially an additive model; the purpose is to continuously optimize the promotion value between the actual value error, and the error value is expressed by a loss function, that is, to minimize the loss function. When the loss function is a square function, each base learner fits the residual of the previous stage; when the loss function is a general function, the core idea of the gradient boosting tree needs to be used.

The NN algorithm based on the neural network (NN) model is divided into two processes, one is the forward propagation of the signal, and the other is the backward feedback of the error. During forward propagation, the input vector is passed in from the input layer and then multiplied by the corresponding 4 weights, and the result is passed to different neurons in the first hidden layer, and then, through all hidden layers and similar processing to the final, the output layer outputs the final value. If it does not match the output value in the training sample, it begins to enter the error reverse feedback stage. We need to use this error value to adj'propagation, the error of the output layer is divided according to the ratio of the link weight, and then, the weight between the hidden layer and the output layer is optimized to make the error function take the minimum value.

\section{Empirical Analysis of the Theory and Technology of Big Data Physical Health Promotion}

4.1. Male Physical Health Data. After determining the research object, according to the adolescent physical data evaluation model method, the male physical fitness measurement data is calculated and processed, and the female measurement data is also applied to the model. As shown in Figure 1, the results of the promotion classification effect of the boys' $50 \mathrm{~m}$ run show that the accuracy of the promotion classification for the boys' $50 \mathrm{~m}$ run is between $77 \%$ and $80 \%$, and when the proportion of the training set is $60 \%$, the accuracy of the three methods reached the highest value. In terms of data processing, as the proportion of training sets increases, the GBDT method has the highest accuracy rate, reaching 79.79\%; in the Macro-F1 index, GBDT is significantly better than the other two methods, so the GBDT method has the best performance. The physique data classification training research data is shown in Table 1.

As shown in Table 2, the average weight of boys aged $17-18$ is $1.97 \mathrm{~kg}$ heavier than average. The difference in 18 years is $4.36 \mathrm{~kg}$, and the difference in 17 years is only $0.10 \mathrm{~kg}$. The average weight of boys aged 17-18 is $4.01 \mathrm{~kg}$ higher than the minimum, and the maximum difference between 18 is $5.99 \mathrm{~kg}$. The average weight of boys aged $17-18$ is $2.03 \mathrm{~kg}$ higher than the minimum, and the average weight of boys aged $17-18$ is more than $3 \mathrm{~kg}$ higher. The maximum difference for 18 years old is $3.79 \mathrm{~kg}$, and the minimum difference is $0.44 \mathrm{~kg}$. Adolescent girls aged 17-18 weigh basically the same as the average, with a mean difference of $0.08 \mathrm{~kg}$.

As shown in Figure 2, the results of the classification effect of promoting forward bending of the male sitting position show that the classification accuracy of promoting forward bending of the male sitting position is between $67 \%$ and $70 \%$, and the accuracy rate of the RF method is always higher than that of the other two. When the set ratio is $50 \%$, the accuracy rate reaches the highest, $69.28 \%$.

As shown in Figure 3, the results of the promotion classification effect of the boys' $1000 \mathrm{~m}$ run show that the accuracy of the promotion classification for the boys' $1000 \mathrm{~m}$ run is between $72 \%$ and $78 \%$. When the proportion of the training set is $80 \%$, the three methods achieve their respective optimal effectiveness, and the RF method achieved the highest promotion accuracy rate of $77.62 \%$; on the Macro-F1 index, GBDT and RF methods have similar effects.

As shown in Table 3, the results of boys pull-ups to promote classification results show that the accuracy of classification for boys pull-ups is between $90 \%$ and $92 \%$. When the proportion of the training set is $60 \%$, the RF method takes the highest accuracy; the rate is $92.04 \%$; on the Macro-F1 index, the GBDT method is obviously the best. 


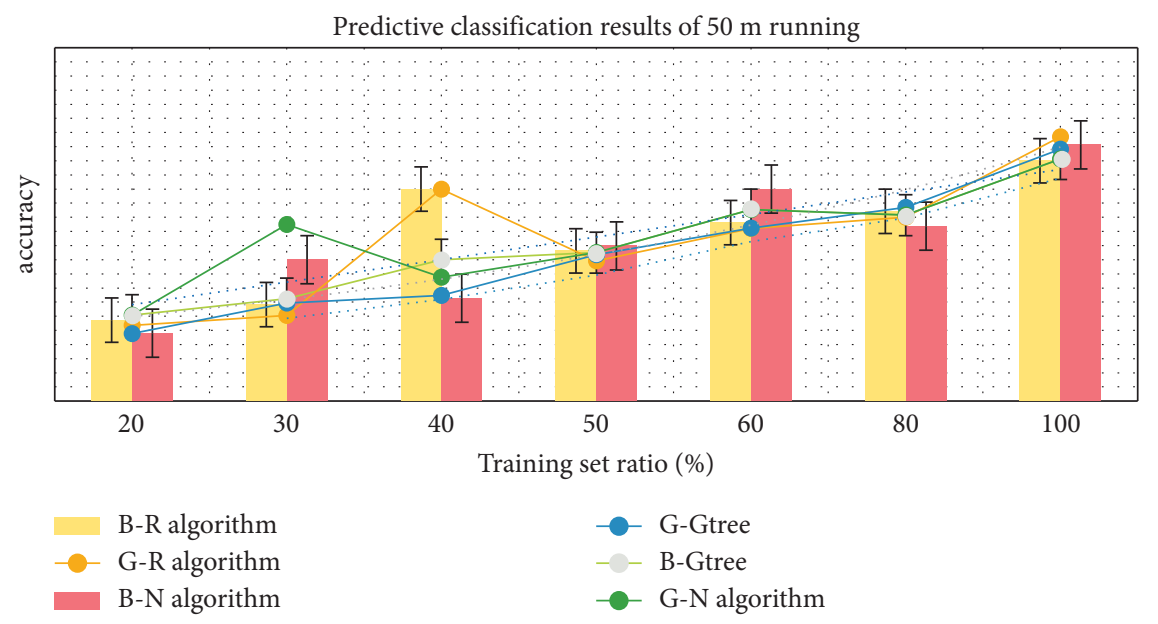

Figure 1: Predictive classification results of $50 \mathrm{~m}$ running.

TABLE 1: Predictive effect of male $50 \mathrm{~m}$ running grade.

\begin{tabular}{|c|c|c|c|c|c|c|}
\hline \multirow[b]{2}{*}{ Training set ratio (\%) } & \multicolumn{3}{|c|}{ Accuracy (\%) } & \multicolumn{3}{|c|}{ Macro-F1 index } \\
\hline & $\begin{array}{l}\text { Random } \\
\text { forest algorithm }\end{array}$ & $\begin{array}{c}\text { Gradient } \\
\text { boosting tree }\end{array}$ & $\begin{array}{c}\text { Neural } \\
\text { network algorithm }\end{array}$ & $\begin{array}{l}\text { Random forest } \\
\text { algorithm }\end{array}$ & $\begin{array}{c}\text { Gradient } \\
\text { boosting tree }\end{array}$ & $\begin{array}{l}\text { Neural network } \\
\text { algorithm }\end{array}$ \\
\hline 20 & 69.14 & 68.89 & 67.91 & 0.428 & 0.360 & 0.225 \\
\hline 30 & 68.60 & 68.56 & 67.66 & 0.409 & 0.354 & 0.242 \\
\hline 40 & 69.17 & 69.32 & 68.40 & 0.421 & 0.379 & 0.255 \\
\hline 50 & 68.99 & 69.54 & 68.50 & 0.439 & 0.360 & 0.274 \\
\hline 60 & 69.21 & 69.79 & 68.83 & 0.440 & 0.371 & 0.279 \\
\hline 80 & 69.09 & 69.72 & 68.72 & 0.422 & 0.354 & 0.289 \\
\hline
\end{tabular}

TABle 2: Physical mass ratio of athletic young boys.

\begin{tabular}{lccr}
\hline Training set ratio (\%) & Accuracy (\%) & Neural network algorithm \\
\hline 20 & Random forest algorithm & Gradient boosting tree & 74.66 \\
30 & 73.92 & 73.70 & 74.93 \\
40 & 73.84 & 73.15 & 73.95 \\
50 & 73.91 & 73.36 & 73.75 \\
60 & 74.10 & 73.85 & 74.56 \\
80 & 75.13 & 74.62 & 75.73 \\
\hline
\end{tabular}

4.2. Female Physical Health Data. As shown in Figure 4, the results of the promotion classification effect of $50 \mathrm{~m}$ running for girls show that the classification accuracy for $50 \mathrm{~m}$ running for girls is between $83 \%$ and $86 \%$. When the proportion of the training set is less than $60 \%$, the RF method is the best, and the highest is $84.67 \%$; the rest are the best GBDT method, and the highest is $85.74 \%$; in the MacroF1 index, the GBDT method is the best. The results of the classification effect for girls standing long jump promotion show that the accuracy rate of classification for girls standing long jump promotion is between $51 \%-56 \%$. When the training set is less than $60 \%$, the RF method is the best, the highest is $53.93 \%$, the rest are GBDT the method is the best, and the highest is $55.46 \%$; in the Macro-F1 index, RF and GBDT have their own advantages.

As shown in Figure 5, the results of the classification effect of female sitting forward bending promotion show that the classification accuracy for female sitting forward bending promotion is between $60 \%$ and $65 \%$, and the accuracy of the NN method is the best. When the training set ratio is $60 \%$, the accuracy is the highest, reaching $64.73 \%$; on the Macro-F1 index, the GBDT method is the best. There are many ways to achieve the curriculum goals, but it is not that the more field goals the better, and the ultimate goal is still to be achieved by physical exercise. The sports items in the physical exercise content all have their own characteristics. Only by teaching youth sports skills, intensive exercises inside and outside the classroom, and applying them in practice, can young people gradually realize the basic characteristics and cultural connotation of this sport. This is what can be achieved as a clear goal.

As shown in Figure 6, the results of the promotion classification effect of $800 \mathrm{~m}$ running for girls show that the classification accuracy for $800 \mathrm{~m}$ running for girls is between $74 \%$ and $79 \%$. The accuracy of the RF method is always the best, and it is accurate when the training set ratio is $80 \%$. The 


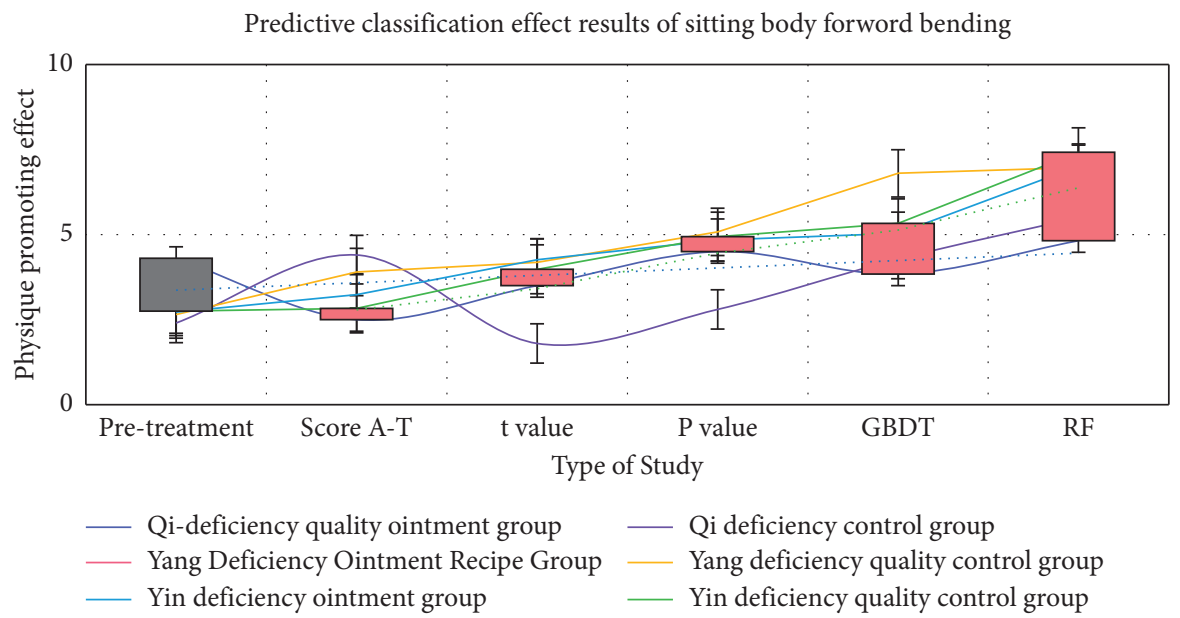

Figure 2: Predictive classification effect results of sitting body forward bending.

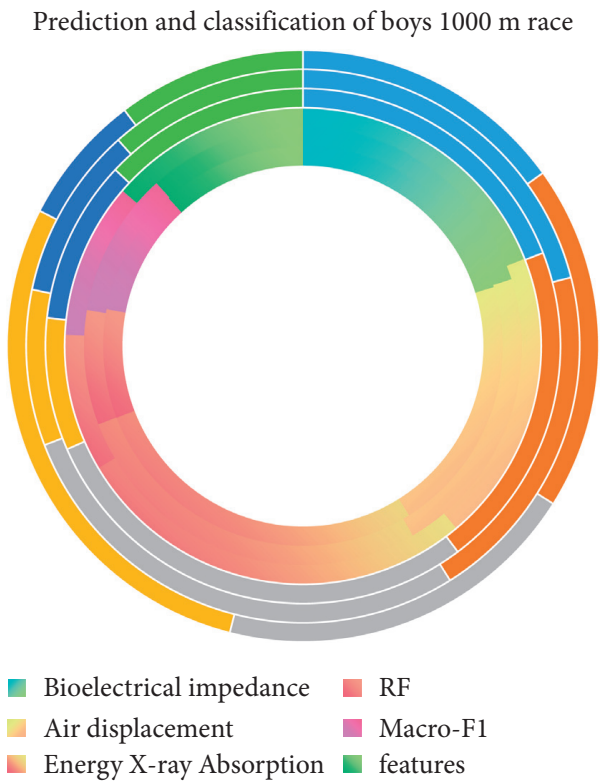

Figure 3: Prediction and classification of boys' 1000m race.

TABle 3: Correlation between BAI/BMI and BIA, ADP, and DXA.

\begin{tabular}{lccr}
\hline Body fat rate measurement method & $r$ & $r^{2}$ & See \\
\hline Bioelectrical impedance (BIA) & $0.87 / 0.90$ & $0.76 / 0.80$ & $2.22 / 2.01$ \\
Air displacement (ADP) & $0.73 / 0.81$ & $0.53 / 0.65$ & $3.99 / 3.45$ \\
Dual energy X-ray absorption (DXA) & $0.42 / 0.65$ & $0.17 / 0.42$ & $8.18 / 6.86$ \\
\hline
\end{tabular}

highest rate, reaching $78.06 \%$; on the Macro-F1 index, the GBDT method is obviously the best.

As shown in Table 4, relatively boring and dangerous sports such as gymnastics and track and field are rejected. The content of health education is not clear due to factors such as exercise methods, teachers, and implementation plans. Many schools have curriculum settings. Nameless and put it on the shelf. This situation has not changed since the implementation of the curriculum standards in the past 20 years. The voice of pushing all responsibilities to the grassroots schools and physical education teachers is questionable, and it also shows that there are still problems to be solved in health education.

As shown in Figure 7, the results of the classification results for girls' 1-minute sit-ups show that the classification accuracy of girls' one-minute sit-ups is between $70 \%$ and $74 \%$. The accuracy of the NN method is always the best. The accuracy rate is the highest at $80 \%$, reaching $73.84 \%$; on the Macro-F1 index, the GBDT method is the best.

As shown in Figure 8, the result of promoting classification effect of girls' final physique level shows that, among the effect of promoting classification of girls' final 


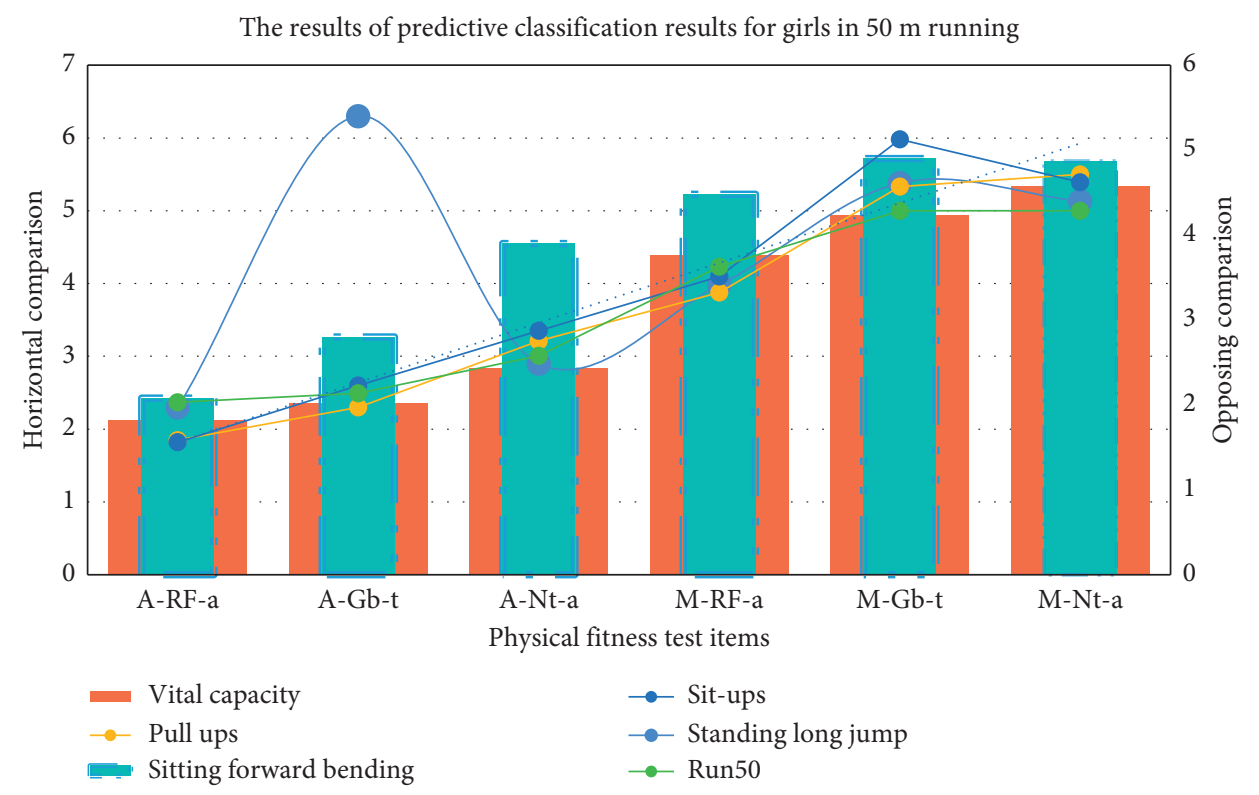

FIGURE 4: The results of predictive classification results for girls in $50 \mathrm{~m}$ running.

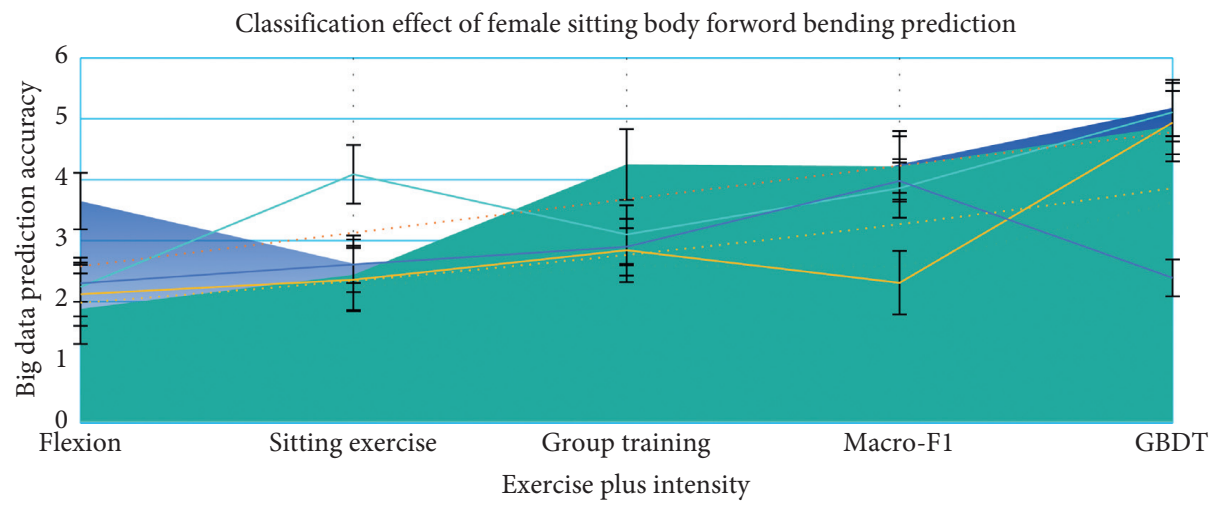

Basic connotation

Physical requirements

_ optimize the target

Project perspective

FIGURE 5: Classification effect of female sitting body forward bending prediction.

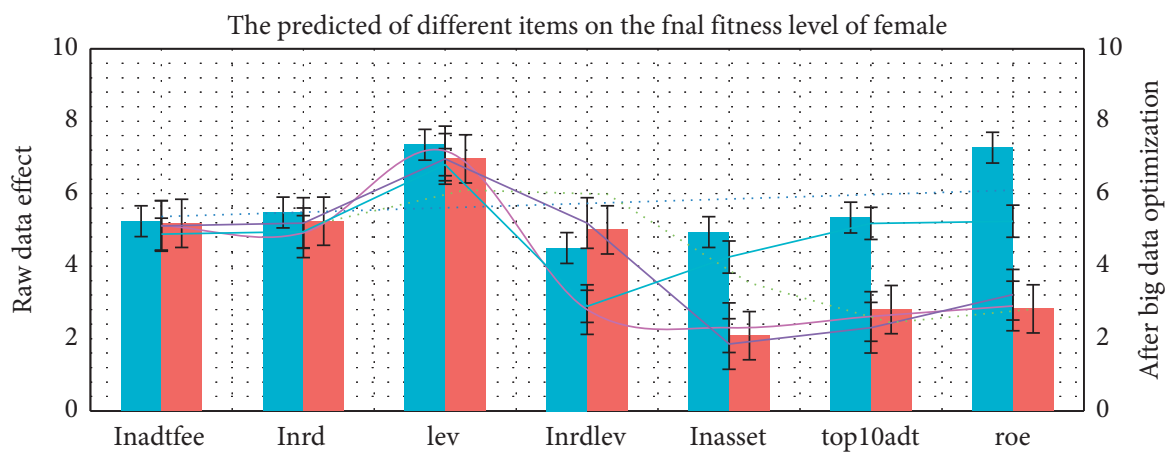

Project definition modulus

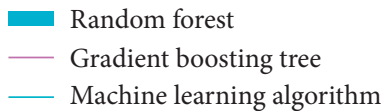

Neural network algorithm

— One Hot Encoding

Figure 6: The predicted performance of different items on the final fitness level of female. 
TABLE 4: Macro-F1 index of female fitness monitoring.

\begin{tabular}{lccc}
\hline Training set ratio (\%) & Macro-F1 index & \\
& Random forest algorithm & Gradient boosting tree & Neural network algorithm \\
\hline 20 & 0.382 & 0.408 & 0.213 \\
30 & 0.394 & 0.423 & 0.213 \\
40 & 0.403 & 0.418 & 0.218 \\
50 & 0.408 & 0.446 & 0.257 \\
60 & 0.390 & 0.429 & 0.228 \\
80 & 0.363 & 0.398 & 0.242 \\
\hline
\end{tabular}

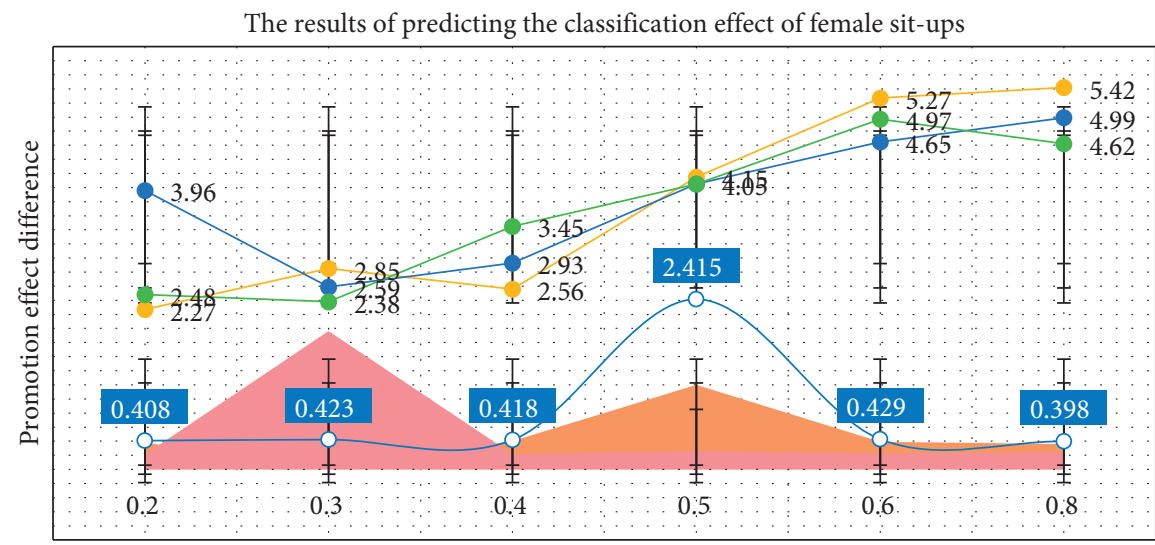

Physical fitness prediction model accuracy

$\begin{array}{ll}\text { Random forest algorithm } & \text { Neural network algorithm } \\ -0-\text { Gradient boosting tree } & - \text { - position } \\ - \text { precise } & - \text { - features }\end{array}$

FIgURE 7: The results of predicting the classification effect of female sit-ups.

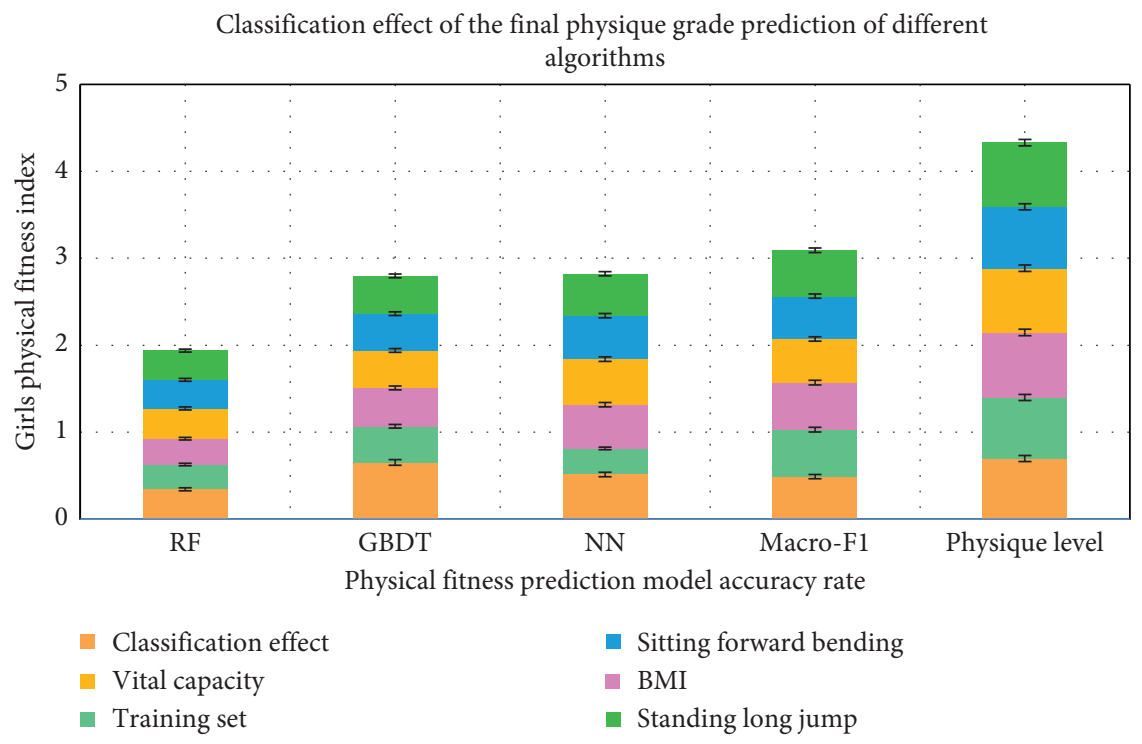

Figure 8: Classification effect of the final physique grade prediction of different algorithms. 
physique level, the accuracy of RF and GBDT methods is between $90 \%$ and $96 \%$, while the accuracy of NN method is $80 \%-87 \%$; when the training set proportion is $80 \%$, the GBDT method achieves the highest accuracy rate of 95.06\%; on the Macro-F1 index, the GBDT method is obviously the best.

\section{Conclusions}

The youth physical health promotion technology has the function of evaluation, promotion, and monitoring. Through the evaluation of test data and the promotion of development trends, countermeasures are formulated to promote specific groups to pay attention to their physical health and strengthen physical exercise. This paper investigates and analyzes the physical health data of adolescents in my country. Physical Health Promotion puts forward the hypothesis that the results of health intervention training as part of the empirical research part of the construction of adolescent health big data management service system can effectively improve the physical health of adolescents.

In the processing of data, GBDT is suitable when the training set is relatively large, and as the sample size increases, the accuracy rate can reach $79.79 \%$. In terms of the classification accuracy of male sitting forward bending promotion, the accuracy of the RF method is higher than that of GBDT. In terms of the promotion classification effect of boys' $1000 \mathrm{~m}$ running, the RF method achieved the highest promotion accuracy rate of $77.62 \%$. In the male pull-ups to promote the classification effect, when the proportion of the training set is $60 \%$, the RF method gets the highest accuracy rate, which is $92.04 \%$. The results of the classification effect for girls standing long jump promotion show that the classification accuracy rate for girls standing long jump promotion is between $51 \%$ and $56 \%$. When the training set is less than $60 \%$, the RF method is the best, the highest is $53.93 \%$, and the rest are GBDT. The method is the best, the highest is $55.46 \%$; in the Macro-F1 index, RF and GBDT have their own advantages. In the promotion of the classification effect on the final fitness level of girls, the accuracy of the RF and GBDT methods is between $90 \%$ and $96 \%$, and the accuracy of the NN method is between $80 \%$ and $87 \%$; when the proportion of the training set is $80 \%$, the GBDT method achieves the highest accuracy rate of $95.06 \%$; on the Macro-F1 index, the GBDT method is obviously the best.

The research sample in this article is limited, and to a certain extent, it cannot reflect the promotion effect of all adolescents' physical health. Although the accuracy of the GBDT algorithm can reach $95 \%$ when the proportion of the dataset is $80 \%$, there may be some training for different samples and different results, and this part remains to be studied.

\section{Data Availability}

The data used to support the findings of this study are available from the corresponding author upon reasonable request.

\section{Conflicts of Interest}

The author declares that there are no conflicts of interest regarding the publication of this paper.

\section{References}

[1] L. Juul, V. J. Andersen, J. Arnoldsen, and H. T. Maindal, "Effectiveness of a brief theory-based health promotion intervention among adults at high risk of type 2 diabetes: oneyear results from a randomised trial in a community setting," Primary Care Diabetes, vol. 10, no. 2, pp. 111-120, 2016.

[2] L. Beaton and R. Freeman, "Oral health promotion and homelessness: a theory-based approach to understanding processes of implementation and adoption," Health Education Journal, vol. 75, no. 2, pp. 184-197, 2016.

[3] M. Borhani, R. Sadeghi, D. Shojaeizadeh, T. Fasihi Harandi, and M. A. Vakili, “Teenage girls' experience of the determinants of physical activity promotion: a theory-based qualitative content analysis," Electronic Physician, vol. 9, no. 8, pp. 5075-5082, 2017.

[4] J. Bnnelycke, C. T. Sandholdt, and A. P. Jespersen, "Household collectives: resituating health promotion and physical activity," Sociology of Health \& Illness, vol. 41, no. 3, pp. 36-39, 2019.

[5] S. J. Winter, J. L. Sheats, and A. C. King, "The use of behavior change techniques and theory in technologies for cardiovascular disease prevention and treatment in adults: a comprehensive review," Progress in Cardiovascular Diseases, vol. 58, no. 6, pp. 605-612, 2016.

[6] V. Burau, K. Carstensen, M. Fredens, and M. B. Kousgaard, "Exploring drivers and challenges in implementation of health promotion in community mental health services: a qualitative multi-site case study using normalization process theory," BMC Health Services Research, vol. 18, no. 1, pp. 36-42, 2018.

[7] S. Tiitinen, S. Ilomki, J. Laitinen et al., "Developing theoryand evidence-based counseling for a health promotion intervention: a discussion paper," Patient Education and Counseling, vol. 103, no. 1, pp. 45-46, 2019.

[8] O. Østerlie, A. Løhre, and G. Haugan, “The expectancy-value questionnaire in physical education: a validation study among Norwegian adolescents," Scandinavian Journal of Educational Research, vol. 63, no. 6, pp. 869-883, 2019.

[9] E. Vogel, "Clinical specificities in obesity care: the transformations and dissolution of "will" and "drives"," Health Care Analysis, vol. 24, no. 4, pp. 321-337, 2016.

[10] S. Hales, G. M. Turner-Mcgrievy, S. Wilcox et al., "Social networks for improving healthy weight loss behaviors for overweight and obese adults: a randomized clinical trial of the social pounds off digitally (Social POD) mobile app," International Journal of Medical Informatics, vol. 94, no. 10, pp. 81-90, 2016.

[11] J. Peng, J. Quan, and L. Peng, "It application maturity, management institutional capability and process management capability," Journal of Organizational and End User Computing, vol. 31, no. 1, pp. 61-85, 2019.

[12] A. B. Mayer, R. J. Mcdermott, C. A. Bryant, J. A. Baldwin, and J. Kromrey, "Sustainability of community-based health promotion coalitions: putting theory into practice," Health Behavior and Policy Review, vol. 4, no. 6, pp. 511-520, 2017.

[13] A. K. Yetisen, A. F. Coskun, G. England et al., "Art on the nanoscale and beyond," Advanced Materials, vol. 28, no. 9, pp. 1724-1742, 2016. 
[14] K. Walters, R. Frost, K. Kharicha et al., "Home-based health promotion for older people with mild frailty: the homehealth intervention development and feasibility RCT," Health Technology Assessment, vol. 21, no. 73, pp. 1-128, 2017.

[15] V. K. Nahar, M. Sharma, H. P. Catalano, M. J. Ickes, P. Johnson, and M. A. Ford, "Testing multi-theory model (MTM) in predicting initiation and sustenance of physical activity behavior among college students," Health Promotion Perspectives, vol. 6, no. 2, pp. 58-65, 2016.

[16] M. Hall and E. Elise, "Theory in chronic disease prevention and health promotion," American Journal of Health Education, vol. 47, no. 4, pp. 202-203, 2016.

[17] D. Sleet, P. Branscum, and A. P. Knowlden, "Advancing theory in health promotion and community health foreword," Family \& Community Health, vol. 40, no. 1, pp. 1-2, 2017.

[18] J. Kim, L. Tao, S.-H. Lee, and J. Diesner, "Evolution and structure of scientific co-publishing network in Korea between 1948-2011," Scientometrics, vol. 107, no. 1, pp. 27-41, 2016.

[19] L. Fabisiak, "Web service usability analysis based on user preferences," Journal of Organizational and End User Computing, vol. 30, no. 4, pp. 1-13, 2018.

[20] L. Ramsey Buchanan, C. R. Rooks-Peck, R. K. C. Finnie et al., "Reducing recreational sedentary screen time," American Journal of Preventive Medicine, vol. 50, no. 3, pp. 402-415, 2016.

[21] K. Silverman, A. F. Holtyn, and B. P. Jarvis, "A potential role of anti-poverty programs in health promotion," Preventive Medicine: An International Journal Devoted to Practice and Theory, vol. 92, no. 2, pp. 58-61, 2016.

[22] D. E. Rosenberg, A. K. Lee, M. Anderson et al., "Reducing sedentary time for obese older adults: protocol for a randomized controlled trial," Jmir Res Protoc, vol. 7, no. 2, pp. 15-23, 2018.

[23] N. Idler, J. Huber, S. Von Mutius, L. Welbergen, and M. R. Fischer, "Prevention and health promotion from theory to practice: the interprofessional mempe summer university for students of medicine, master of public health and epidemiology," GMS Journal for Medical Education, vol. 33, no. 5, 2016.

[24] R. D. Renard, "Creating the other requires defining Thainess against which the other can exist: early-twentieth century definitions (Redefining"otherness"from Northern Thailand)," Tonan Ajia Kenkyu, vol. 44, no. 3, pp. 295-320, 2017.

[25] F. Venetsanou and A. Kambas, "Physical activity promotion in Greek preschools: the gap between theory and practice," Early Childhood Education Journal, vol. 45, no. 3, pp. 437444, 2017.

[26] K. G. Srinivasa, B. J. Sowmya, A. Shikhar, R. Utkarsha, and A. Singh, "Data analytics assisted internet of things towards building intelligent healthcare monitoring systems: iot for healthcare," Journal of Organizational and End User Computing, vol. 30, no. 4, pp. 83-103, 2018.

[27] M. Ssm, N. R. Tonekaboni, R. Farmanbar et al., "The effect of motivational interviewing-based intervention using self-determination theory on promotion of physical activity among women in reproductive age: a randomized clinical trial," Electronic Physician, vol. 9, no. 5, pp. 4461-4472, 2017. 